Canad. J. Math. Vol. 63 (1), 2011 pp. 136-152

doi:10.4153/CJM-2010-078-9

(C) Canadian Mathematical Society 2010

\title{
Transcendental Nature of Special Values of $L$-Functions
}

\author{
Sanoli Gun, M. Ram Murty, and Purusottam Rath
}

Abstract. In this paper, we study the non-vanishing and transcendence of special values of a varying class of $L$-functions and their derivatives. This allows us to investigate the transcendence of Petersson norms of certain weight one modular forms.

\section{Introduction}

The algebraic nature of special values of $L$-functions is shrouded in mystery. The $L$-functions arise from various contexts like algebraic number theory (Riemann zeta function, Dirichlet $L$-functions, Dedekind zeta functions, $L$-series associated with Hecke grossencharacters), representation theory, and theory of automorphic forms (Artin $L$-functions, Rankin-Selberg $L$-functions of classical modular forms and their generalisations to Hilbert, and Siegel modular forms and to automorphic forms on more complicated groups) and algebraic geometry (zeta-functions of varieties over number fields). For all such $L$-functions, it is believed that the special values of such $L$-functions should be, up to an algebraic factor, equal to a predictable period. We refer to the article of Zagier [21] for further motivations.

However, barring a very few special cases, our understanding of these special values is rather limited. For $L$-functions associated with classical Dirichlet characters, we have had some success thanks to the remarkable theorem of Baker on transcendence of linear forms in logarithms of algebraic numbers (see [1] or [13]). More precisely, we know that $L(1, \chi)$ is transcendental for any non-trivial Dirichlet character $\chi$. In fact, it is expressible as an algebraic linear combination of logarithms of algebraic numbers. However, as soon as we consider the Dedekind zeta functions or, more generally, special values of Artin $L$-series, we run into difficulty.

The theme of our present work is to investigate the non-vanishing of a varying class of $L$-functions as well as their derivatives, mainly at the point $s=1$. In such cases, we also study the algebraic nature of these $L$-values. Also, where the $L$-function has a pole at $s=1$, as for Dedekind zeta functions and certain Artin $L$-functions, we wish to investigate the algebraic nature of the residue at that point.

We begin our work by enlisting some transcendental prerequisites essential for our work. We do this in the next section. We derive an important consequence of a conjecture of Schanuel. This is central to investigating the special values of Dedekind zeta functions as well as Artin $L$-functions. Admittedly, this is a strong conjecture,

Received by the editors August 18, 2008; revised January 20, 2009.

Published electronically November 6, 2010.

M. R. Murty's research partially supported by Natural Sciences and Engineering Research Council.

AMS subject classification: 11J81, 11J86, 11 J91. 
but it does suggest that the transcendence of $L(1, \chi)$, where $\chi$ is a non-trivial Dirichlet character, is not an isolated phenomenon and perhaps is a very special case of a general transcendence theorem embracing a wide class of $L$-functions. As our investigation suggests, a proof of Schanuel's conjecture will establish the transcendence of special values of a plethora of $L$-functions.

In Section three, we consider $L$-functions that are generalisations of the classical Dirichlet $L$-functions. We consider the non-vanishing as well as the algebraic nature of derivatives of such $L$-functions $L^{\prime}(s, f)$, where $f$ is a Dirichlet-type function at both $s=0$ as well as $s=1$. For instance, we prove the following.

Theorem 1.1 If $f$ is an even Dirichlet-type periodic function which takes algebraic values, then $L^{\prime}(0, f)$ is either zero or transcendental.

For odd functions, a conjecture of Lang about the independence of gamma values is relevant. We suggest the following conjecture which is a variant of his conjecture.

Conjecture 1.2 For any positive integer $q>2$, let $\overline{V_{\Gamma}(q)}$ denote the vector space over (0) spanned by the real numbers

$$
\log \Gamma(a / q), \quad 1 \leq a \leq q, \quad(a, q)=1 .
$$

Then the dimension of $\overline{V_{\Gamma}(q)}$ is $\varphi(q)$.

Then we have the following.

Theorem 1.3 Let $q>2$. Assume that the above conjecture is true. Then $L^{\prime}(1, \chi)=0$ for at most one primitive odd character mod q.

In the next section, we consider $L$-functions associated with different classes of modular forms, primarily cuspidal eigenforms. We establish various non-vanishing as well as transcendence results. For instance, we prove the following.

Theorem 1.4 Let $f$ be a normalized cuspidal eigenform of weight $2 k$ for $\Gamma_{0}(N)$. Assume that $L(k, f) \neq 0$. Then one has

$$
\frac{L^{\prime}(k, f)}{L(k, f)}=-\psi(k)+\log 2 \pi-\frac{1}{2} \log N,
$$

where $\psi$ is the logarithmic derivative of the gamma function. Further, for such an $f$, $L^{\prime}(k, f) \neq 0$.

We derive various consequences of the above theorem. For instance, we have the following.

Corollary 1.5 Let $E$ be an elliptic curve over $(\mathbb{O}$. Suppose that $L(1, E) \neq 0$. Then $L^{\prime}(1, E) \neq 0$.

We also derive various transcendence results for special values of $L$-functions and symmetric square $L$-functions associated to modular forms. 
In the penultimate section, we begin by considering Dedekind zeta functions. We show that the regulator of a number field is transcendental under a weaker version of the conjecture of Schanuel. We refer to the next section for the statement of the Weak Schanuel Conjecture. We also show that the residue of the Dedekind zeta function at $s=1$ is also transcendental, again under the conjecture of Schanuel. Then more generally, we consider the special values of Artin $L$-functions motivated by the work of Stark. We have the following result.

Theorem 1.6 Assume that the Weak Schanuel Conjecture is true. Then for any rational non-trivial irreducible character $\chi, L(1, \chi, K / k)$ is transcendental.

Finally, the work of Stark also allows us to interpret these in terms of the transcendence of the Petersson norms of special classes of modular forms of weight one. This motivated us to consider the possible transcendence of norms of such modular forms. For instance, we prove the following.

Theorem 1.7 Let $f$ be a normalised weight 1 Hecke eigen cusp form on $\Gamma_{0}(N)$ with nebentypus $\epsilon$. Suppose $f$ has rational coefficients. Then its Petersson norm $\langle f, f\rangle$ is transcendental under the Weak Schanuel Conjecture.

In some special cases, we can deduce the transcendence of such Petersson norms unconditionally.

\section{Transcendental Requisites}

The most important ingredient for many of our results is the following theorem due to Baker. Let $\mathcal{L}$ denote the set of logarithms of non-zero algebraic numbers. Then we have the following.

Theorem 2.1 If $\lambda_{1}, \lambda_{2}, \ldots, \lambda_{n}$ are $(\mathrm{O}$-linearly independent elements of $\mathcal{L}$, then $1, \lambda_{1}, \lambda_{2}, \ldots, \lambda_{n}$ are $(\overline{\mathrm{O}})$-linearly independent.

An immediate consequence of the above theorem is that any non-zero element in the $\overline{\mathbb{O}}$ vector space

$$
\left\{\alpha_{1} \lambda_{1}+\cdots+\alpha_{n} \lambda_{n} \mid n \in \mathbb{N}, \quad \alpha_{i} \in \overline{\mathbb{O} 2}, \quad \lambda_{i} \in \mathcal{L}\right\}
$$

is necessarily transcendental. Following [13], the elements of this vector space will be called a Baker period. Thus a non-zero Baker period is transcendental.

On the other hand, the question of algebraic independence of transcendental numbers or even more specifically those of numbers connected with the exponential function is rather delicate. One of the very few general results is the following classical result due to Lindemann and Weierstrass [19].

Theorem 2.2 If $\beta_{1}, \ldots, \beta_{n}$ are algebraic numbers that are linearly independent over $(0)$, then the numbers $e^{\beta_{1}}, \ldots, e^{\beta_{n}}$ are algebraic independent.

The most far-reaching conjecture in this set-up is the following, due to Schanuel ([10]). 
Schanuel's Conjecture Suppose $\alpha_{1}, \ldots, \alpha_{n}$ are complex numbers that are linearly independent over $(\mathbb{O}$. Then the transcendence degree of the field

$$
\mathbb{Q}\left(\alpha_{1}, \ldots, \alpha_{n}, e^{\alpha_{1}}, \ldots, e^{\alpha_{n}}\right)
$$

over $(\mathbb{Q})$ is at least $n$.

This conjecture is believed to include all known transcendence results as well as all reasonable transcendence conjectures on the values of the exponential function. We will need the following special case of Schanuel's conjecture.

Weak Schanuel Conjecture Let $\alpha_{1}, \ldots, \alpha_{n}$ be non-zero algebraic numbers such that $\log \alpha_{1}, \ldots, \log \alpha_{n}$ are linearly independent over $\mathbb{Q}$. Then these numbers are algebraically independent.

We shall need the following important consequence of the Weak Schanuel Conjecture.

Proposition 2.3 Assume the Weak Schanuel Conjecture. Let $\alpha_{1}, \cdots, \alpha_{n}$ be non-zero algebraic numbers. Then for any polynomial $f\left(x_{1}, \cdots, x_{n}\right)$ with algebraic coefficients such that $f(0, \cdots, 0)=0, f\left(\log \alpha_{1}, \cdots, \log \alpha_{n}\right)$ is either zero or transcendental.

Proof We use induction on $n$. For $n=1$, the lemma is true by the classical HermiteLindemann theorem. Suppose $f\left(x_{1}, \ldots, x_{n}\right) \in \overline{\mathbb{O}}\left[x_{1}, \ldots, x_{n}\right], n \geq 2$ such that $A:=$ $f\left(\log \alpha_{1}, \ldots, \log \alpha_{n}\right)$ is algebraic. By the Weak Schanuel Conjecture, the numbers $\log \alpha_{1}, \ldots, \log \alpha_{n}$ are linearly dependent over $\left(\mathbb{O}\right.$. Then there exist integers $c_{1}, \ldots, c_{n}$ such that

$$
c_{1} \log \alpha_{1}+\cdots+c_{n} \log \alpha_{n}=0 .
$$

Suppose $c_{1} \neq 0$. Then $\log \alpha_{1}=\frac{1}{c_{1}}\left(c_{2} \log \alpha_{2}+\cdots+c_{n} \log \alpha_{n}\right)$. Replacing this value of $\log \alpha_{1}$ in the expression for $A$, we have

$$
A=g\left(\log \alpha_{2}, \ldots, \log \alpha_{n}\right),
$$

where $g\left(x_{1}, \ldots, x_{n-1}\right)$ is a polynomial with algebraic coefficients in $n-1$ variables. Then by induction hypothesis, $A=0$. This completes the proof of the lemma.

An immediate consequence of Schanuel's conjecture is that for a non-zero algebraic number $\alpha$, the two numbers $e^{\alpha}$ and $\pi$ are algebraically independent and so are the numbers $\log \alpha$ and $\log \pi$. We refer to [7] for some other applications of Schanuel's conjecture.

\section{$3 \quad$ L-Functions of Arbitrary Dirichlet-Type Periodic Functions}

Here we consider $L$-functions that are generalisations of the classical Dirichlet $L$-functions. Let $f$ be any periodic arithmetic function with period $q>1$ such that

$$
f(a) \in \overline{\mathbb{O}} \text { for }(a, q)=1 \quad \text { and } \quad f(a)=0 \text { for }(a, q)>1 .
$$


We call such a function a Dirichlet-type function. Associated with such a function, we have

$$
L(s, f):=\sum_{n=1}^{\infty} \frac{f(n)}{n^{s}}=\sum_{a=1}^{q} f(a) \sum_{n=0}^{\infty} \frac{1}{(a+q n)^{s}}=q^{-s} \sum_{a=1}^{q} f(a) \zeta(s, a / q),
$$

where $\zeta(s, x)$ is the Hurwitz zeta function defined by

$$
\zeta(s, x)=\sum_{n=0}^{\infty} \frac{1}{(n+x)^{s}}, \quad 0<x \leq 1
$$

When $x=1$, we retrieve the familiar Riemann zeta function. The Hurwitz zeta function extends meromorphically to the entire complex plane with a simple pole at $s=1$, where it has a residue 1 . We also have the following interesting identities due to Lerch [11]:

$$
\zeta(0, a / q)=\frac{1}{2}-a / q, \quad \zeta^{\prime}(0, a / q)=\log \Gamma(a / q)-\frac{1}{2} \log 2 \pi .
$$

We note that the series $\sum_{n=1}^{\infty} \frac{f(n)}{n}$ converges if and only if $\sum_{a=1}^{q} f(a)=0$ and in which case (see [13, Theorem 16]),

$$
L(1, f)=\frac{-1}{q} \sum_{a=1}^{q} f(a) \psi(a / q)
$$

Here we consider the derivative of the function $L(s, f)$. Interestingly, when $f$ is an even function, the existence of a pole at $s=1$ does not preclude us from concluding the nature of $L^{\prime}(0, f)$. More precisely, we have the following.

Theorem 3.1 If $f$ is an even Dirichlet-type periodic function that takes algebraic values, then $L^{\prime}(0, f)$ is either zero or transcendental.

Proof For $\operatorname{Re}(s)>1$, we have

$$
L^{\prime}(s, f)=\sum_{n=1}^{\infty} \frac{-f(n) \log n}{n^{s}}=\frac{-\log q}{q^{s}} \sum_{a=1}^{q} f(a) \zeta(s, a / q)+\frac{1}{q^{s}} \sum_{a=1}^{q} f(a) \zeta^{\prime}(s, a / q) .
$$

Using the identities in (3.1), we derive

$L^{\prime}(0, f)=-\log q \sum_{a=1}^{q} f(a)(1 / 2-a / q)+\sum_{a=1}^{q} f(a) \log \Gamma(a / q)-\frac{1}{2} \log (2 \pi) \sum_{a=1}^{q} f(a)$. 
Since $f$ is even, we have $f(a)=f(q-a)$ and hence

$$
\begin{aligned}
L^{\prime}(0, f)= & -\log q \sum_{a=1}^{q} f(a)(1 / 2-a / q)+\sum_{a=1}^{[q / 2]} f(a)\{\log \Gamma(a / q)+\log \Gamma(1-a / q)\} \\
& -\log (2 \pi) \sum_{a=1}^{[q / 2]} f(a) \\
= & -\log q \sum_{a=1}^{q} f(a)(1 / 2-a / q)+\sum_{a=1}^{[q / 2]} f(a)\left\{\log \pi-\log \sin \left(\frac{\pi a}{q}\right)\right\} \\
& -\log (2 \pi) \sum_{a=1}^{[q / 2]} f(a) .
\end{aligned}
$$

Here we have used the fact that $\Gamma(x) \Gamma(1-x)=\pi / \sin (\pi x)$. Finally, we have the following expression for $L^{\prime}(0, f)$ :

$$
L^{\prime}(0, f)=-\log q \sum_{a=1}^{q} f(a)(1 / 2-a / q)-\sum_{a=1}^{[q / 2]} f(a) \log \sin \left(\frac{\pi a}{q}\right)-\log 2 \sum_{a=1}^{[q / 2]} f(a) .
$$

The right-hand side is an algebraic linear combination of logarithms of algebraic numbers, i.e., a Baker period and hence is either zero or transcendental.

For even primitive Dirichlet characters, we have the following.

Corollary 3.2 If $\chi$ is an even primitive Dirichlet character modulo $q$, then $L^{\prime}(0, \chi)$ is transcendental.

Proof For a primitive even Dirichlet character, the functional equation is

$$
L(s, \chi)=\frac{1}{\pi}\left(\frac{2 \pi}{q}\right)^{s} \Gamma(1-s) S(\chi) \sin \left(\frac{\pi s}{2}\right) L(1-s, \bar{\chi}),
$$

where $S(\chi)=\sum_{a=1}^{q} \chi(a) e^{2 \pi i a / q}$ is a non-zero algebraic number. Taking the derivative and evaluating at $s=0$, we have $L^{\prime}(0, \chi)=\frac{1}{2} S(\chi) L(1, \bar{\chi})$, and the non-vanishing of $L(1, \bar{\chi})$ gives the corollary.

For an even primitive Dirichlet character $\chi$ modulo $q$, it is of interest to consider the non-vanishing of $L^{\prime}(1, \chi)$. For $q>1$, let $Z_{q}$ be the following set

$$
Z_{q}:=\left\{\alpha: \alpha=\frac{L^{\prime}(1, \chi)}{L(1, \chi)} \text { for some even primitive character } \chi \bmod q\right\} .
$$

It will be of interest to show that for all such $q$, the set $Z_{q}$ does not contain 0 . Presumably, $Z_{q} \cap \overline{\mathbb{O} 2}=\varnothing$.

When $\chi$ is an odd character, the recipe followed above for even characters does not work. For $q>1$, we can consider the analogous set $Z_{q}$ defined for odd primitive characters. An important ingredient for studying this set is the following conjecture due to Lang. This is a generalisation of an earlier conjecture due to Rohrlich. 
Conjecture 3.3 (Rohrlich-Lang) For any integer $q>2$, the extension of $(\mathrm{O})$ generated by the set

$$
\{\pi\} \cup\{\Gamma(a / q): 1 \leq a \leq q, \quad(a, q)=1\}
$$

has transcendence degree $1+\varphi(q) / 2$.

We suggest the following variant of the Rohrlich-Lang conjecture.

Conjecture 3.4 For any positive integer $q>2$, let $\overline{V_{\Gamma}(q)}$ denote the vector space over $\overline{(\mathbb{O})}$ spanned by the real numbers

$$
\log \Gamma(a / q), \quad 1 \leq a \leq q, \quad(a, q)=1
$$

Then the dimension of $\overline{V_{\Gamma}(q)}$ is $\varphi(q)$.

For recent developments related to this conjecture, see [8, Theorem 4]. We have the following theorem.

Theorem 3.5 Let $q>2$. Assume that the above variant of Rohrlich-Lang conjecture is true. Then $L^{\prime}(1, \chi)=0$ for at most one odd primitive character mod $q$.

Proof Let $\chi$ be a primitive odd character $\bmod q$. For such a character, the functional equation for $L(s, \chi)$ is

$$
L(s, \chi)=\frac{1}{\pi i}\left(\frac{2 \pi}{q}\right)^{s} \Gamma(1-s) S(\chi) \cos \left(\frac{\pi s}{2}\right) L(1-s, \bar{\chi}) .
$$

Taking the logarithmic derivative, we get

$$
\frac{L^{\prime}(s, \chi)}{L(s, \chi)}=\log \left(\frac{2 \pi}{q}\right)-\psi(1-s)-\frac{\pi}{2} \tan \left(\frac{\pi s}{2}\right)-\frac{L^{\prime}(1-s, \bar{\chi})}{L(1-s, \bar{\chi})}
$$

and hence

$$
\frac{L^{\prime}(0, \chi)}{L(0, \chi)}=\log \left(\frac{2 \pi}{q}\right)+\gamma-\frac{L^{\prime}(1, \bar{\chi})}{L(1, \bar{\chi})}
$$

Using the identities of Lerch and the orthogonality of characters, we have

$$
L^{\prime}(0, \chi)=-\log q \sum_{a=1}^{q} \chi(a)(1 / 2-a / q)+\sum_{a=1}^{q} \chi(a) \log \Gamma(a / q)
$$

Further,

$$
L(0, \chi)=\sum_{a=1}^{q} \chi(a) \zeta(0, a / q)=\frac{-1}{q} \sum_{a=1}^{q} a \chi(a)
$$


is a non-zero algebraic number. Using (3.2) and (3.3), we have

$$
\begin{aligned}
& \frac{L^{\prime}(1, \chi)}{L(1, \chi)}= \\
& \quad \gamma+\log (2 \pi / q)+\frac{\log q}{L(0, \bar{\chi})} \sum_{a=1}^{q} \bar{\chi}(a)(1 / 2-a / q)-\frac{1}{L(0, \bar{\chi})} \sum_{a=1}^{q} \bar{\chi}(a) \log \Gamma(a / q),
\end{aligned}
$$

which simplifies to

$$
\frac{L^{\prime}(1, \chi)}{L(1, \chi)}=\gamma+\log (2 \pi)-\frac{1}{L(0, \bar{\chi})} \sum_{a=1}^{q} \bar{\chi}(a) \log \Gamma(a / q) .
$$

Suppose that for two different odd primitive characters $\chi_{1}$ and $\chi_{2}$ modulo $q$, we have

$$
\frac{L^{\prime}\left(1, \chi_{1}\right)}{L\left(1, \chi_{1}\right)}=0=\frac{L^{\prime}\left(1, \chi_{2}\right)}{L\left(s, \chi_{2}\right)}
$$

This implies that

$$
\sum_{a=1}^{q} \frac{\bar{\chi}_{1}(a)}{L\left(0, \bar{\chi}_{1}\right)} \log \Gamma(a / q)=\sum_{a=1}^{q} \frac{\bar{\chi}_{2}(a)}{L\left(0, \bar{\chi}_{2}\right)} \log \Gamma(a / q) .
$$

Then our conjecture will imply that for all $(a, q)=1$,

$$
\frac{\bar{\chi}_{1}(a)}{L\left(0, \bar{\chi}_{1}\right)}=\frac{\bar{\chi}_{2}(a)}{L\left(0, \bar{\chi}_{2}\right)}
$$

Since $\bar{\chi}_{1}(1)=\bar{\chi}_{2}(1)$, we have $L\left(0, \bar{\chi}_{1}\right)=L\left(0, \bar{\chi}_{2}\right)$ and hence $\chi_{1}=\chi_{2}$.

\section{L-Functions of Modular Forms}

Our purpose in this section is to study $L$-functions and symmetric square $L$-functions associated with modular forms. Let $k, N \geq 1$ be natural numbers. Also let $f=$ $\sum_{n \geq 1} a(n) q^{n}$ be a normalized cuspidal eigenform of weight $2 k$ for $\Gamma_{0}(N)$ and let

$$
L(s, f)=\sum_{n \geq 1} \frac{a(n)}{n^{s}}, \quad s \in \mathbb{C}, \quad \Re(s)>k+1
$$

denote the $L$-function associated with $f$. Recall that

$$
\Lambda_{N}(s):=N^{s / 2}(2 \pi)^{-s} \Gamma(s) L(s, f)
$$

has a holomorphic continuation to the entire complex plane and satisfies the functional equation

$$
\Lambda_{N}(s)= \pm \Lambda_{N}(2 k-s) .
$$

Here, we prove the following. 
Theorem 4.1 Let $f$ be a normalized cuspidal eigenform of weight $2 k$ for $\Gamma_{0}(N)$. Assume that $L(k, f) \neq 0$. Then one has

$$
\frac{L^{\prime}(k, f)}{L(k, f)}=-\psi(k)+\log 2 \pi-\frac{1}{2} \log N
$$

Further, $L^{\prime}(k, f) \neq 0$.

Proof By (4.1), we have

$$
N^{s / 2}(2 \pi)^{-s} \Gamma(s) L(s, f)= \pm N^{(2 k-s) / 2}(2 \pi)^{-2 k+s} \Gamma(2 k-s) L(2 k-s, f) .
$$

Taking the logarithmic derivative with respect to $s$, we see that

$$
\log N+\psi(s)+\frac{L^{\prime}(s, f)}{L(s, f)}=2 \log 2 \pi-\psi(2 k-s)-\frac{L^{\prime}(2 k-s, f)}{L(2 k-s, f)} .
$$

Since $L(k, f) \neq 0$, putting $s=k$ in (4.3), we get the first part of the theorem. To prove the second part of the theorem, note that

$$
-\psi(k)=\gamma-\sum_{n=1}^{k-1} \frac{1}{n}, \quad \text { where } \quad 0.577215<\gamma<0.577216
$$

Now if

$$
\frac{L^{\prime}(k, f)}{L(k, f)}=0
$$

then by (4.4), we will have

$$
\gamma+\log 2 \pi=\sum_{n=1}^{k-1} \frac{1}{n}+\frac{1}{2} \log N
$$

We will show that (4.5) can never happen. Since

$$
2.41421<\gamma+\log 2 \pi<2.415116, \quad 2.418<\frac{1}{2} \log 126 \quad \text { and } \quad \sum_{n=1}^{6} \frac{1}{n}=2.45
$$

we obtain that

$$
\gamma+\log 2 \pi<\sum_{n=1}^{k-1} \frac{1}{n}+\frac{1}{2} \log N
$$

is true for all pairs $(k, N)$, where either $k \geq 7$ or $N \geq 126$. For the remaining cases, that is, when $k \leq 6$ and $N \leq 126$, one can similarly check that 4.5 can never happen.

As immediate corollaries, we have the following. 
Corollary 4.2 Let $f$ be as in the previous theorem. Then

$$
\exp \left(\frac{L^{\prime}(k, f)}{L(k, f)}+\psi(k)\right)
$$

is transcendental.

Proof The corollary follows from equation (4.2).

Corollary 4.3 Let $k \geq 1$ be a fixed natural number. For an $N \geq 1$, let $E(N)$ denote the set of all normalized cuspidal eigenforms $f$ of weight $2 k$ and level $N$ such that $L(k, f) \neq$ 0 . Then the infinite set

$$
\left\{\frac{L^{\prime}(k, f)}{L(k, f)}: f \in E(N), N \geq 1\right\}
$$

has at most one algebraic element.

Proof We note that since $f_{1}, f_{2}$ have the same level $N$, by (4.2),

$$
\frac{L^{\prime}\left(k, f_{1}\right)}{L\left(k, f_{1}\right)}=\frac{L^{\prime}\left(k, f_{2}\right)}{L\left(k, f_{2}\right)}
$$

Hence, we only need to consider forms corresponding to different levels. Let $f_{1} \in$ $E\left(N_{1}\right)$ and $f_{2} \in E\left(N_{2}\right)$, where $N_{1} \neq N_{2}$. Now if both $\frac{L^{\prime}\left(k, f_{1}\right)}{L\left(k, f_{1}\right)}$ and $\frac{L^{\prime}\left(k, f_{2}\right)}{L\left(k, f_{2}\right)}$ are algebraic, then their difference $\frac{1}{2} \log \frac{N_{2}}{N_{1}}$ is algebraic, a contradiction.

Corollary 4.4 Assume Schanuel's conjecture is true. Then

$$
\frac{L^{\prime}(k, f)}{L(k, f)}-\gamma \quad \text { and } \quad \exp \left(\frac{L^{\prime}(k, f)}{L(k, f)}-\gamma\right)
$$

are transcendental.

Proof We have

$$
\begin{gathered}
\frac{L^{\prime}(k, f)}{L(k, f)}-\gamma=\log 2 \pi-\frac{1}{2} \log N-\alpha \text { and } \\
\exp \left(\frac{L^{\prime}(k, f)}{L(k, f)}-\gamma\right)=e^{-\alpha} \frac{2 \pi}{N^{\frac{1}{2}}}, \quad \text { where } \alpha=\sum_{n=1}^{k-1} \frac{1}{n} \in(\mathbb{2})
\end{gathered}
$$

Since by Schanuel's conjecture $\pi$ and any non-zero algebraic power of $e$ are algebraically independent, both the elements are transcendental.

Let $E$ be an elliptic curve defined over $(0)$ with conductor $N$. Since every elliptic curve over $(\mathbb{O})$ is modular (see $[3,4,6,16,20]$ ), we have the following corollaries. 
Corollary 4.5 Let $E$ be an elliptic curve defined over $(\mathbb{O})$ of conductor $N$. Suppose that $L(1, E) \neq 0$. Then $L^{\prime}(1, E) \neq 0$. Further,

$$
\exp \left(\frac{L^{\prime}(1, E)}{L(1, E)}-\gamma\right)
$$

is transcendental.

Corollary 4.6 Let

$$
S:=\left\{\frac{L^{\prime}(1, E)}{L(1, E)}: \text { E an elliptic curve over }(\mathbb{O}, \quad L(1, E) \neq 0\} .\right.
$$

Then S has at most one algebraic element.

Finally, we consider the symmetric square $L$-function associated with a normalized cuspidal eigenform. For a normalized cuspidal eigenform $f=\sum_{n>1} a(n) q^{n}$ of weight $2 k$ for $\Gamma_{0}(1)$, we denote by

$$
L\left(s, S y m^{2} f\right)=\prod_{p}\left(1-\alpha_{p}^{2} p^{-s}\right)^{-1}\left(1-\alpha_{p} \beta_{p} p^{-s}\right)^{-1}\left(1-\beta_{p}^{2} p^{-s}\right)^{-1}, \quad \Re(s)>2 k
$$

the symmetric square $L$-function of $f$, where the product is over all primes $p$ and $\alpha_{p}, \beta_{p}$ are defined by $\alpha_{p}+\beta_{p}=a(p), \alpha_{p} \beta_{p}=p^{2 k-1}$. Recall that $L\left(s, S y m^{2} f\right)$ has a holomorphic continuation (see [15]) to $\mathbb{C}$, and the function

$$
L^{*}\left(s, \operatorname{Sym}^{2} f\right)=2^{-s} \pi^{-3 s / 2} \Gamma(s) \Gamma\left(\frac{s-2 k+2}{2}\right) L\left(s, \operatorname{Sym}^{2} f\right)
$$

satisfies the functional equation

$$
L^{*}\left(s, S y m^{2} f\right)=L^{*}\left(4 k-1-s, S y m^{2} f\right) .
$$

Here we prove the following.

Theorem 4.7 Let $f$ be a normalized cuspidal eigenform of weight $2 k$ for $\Gamma_{0}(1)$. Assume that $L\left(2 k-1 / 2, S_{y m}^{2} f\right) \neq 0$. Then one has

$$
\frac{L^{\prime}\left(2 k-1 / 2, S y m^{2} f\right)}{L\left(2 k-1 / 2, S^{2} m^{2} f\right)}=\log 2 \pi^{3 / 2}-\psi(2 k-1 / 2)-\frac{1}{2} \psi(3 / 4) .
$$

Further, $L^{\prime}\left(2 k-1 / 2, \operatorname{Sym}^{2} f\right) \neq 0$ for all $k>48$.

Proof Taking the logarithmic derivative with respect to $s$ in (4.6), one proves the first part of the theorem. Since

$$
\psi(2 k-1 / 2)=-\gamma-2 \log 2+\sum_{n=1}^{2 k-1} \frac{1}{n-1 / 2},
$$


therefore

$$
\frac{L^{\prime}\left(2 k-1 / 2, S^{2} m^{2} f\right)}{L\left(2 k-1 / 2, S^{2} f\right)}=\frac{3}{2} \gamma+\frac{3}{2} \log \pi+\frac{9}{2} \log 2-\frac{\pi}{4}-\sum_{n=1}^{2 k-1} \frac{1}{n-1 / 2} .
$$

We know

$$
\frac{3}{2} \gamma+\frac{3}{2} \log \pi+\frac{9}{2} \log 2-\frac{\pi}{4}<4.93
$$

and since

$$
\sum_{n=1}^{2 k-1} \frac{1}{n-1 / 2}>4.93
$$

for all $k>48$, 4.7) is non-zero for all such $k$.

Corollary 4.8 Let $f$ be as in the previous theorem. Then one has that

$$
\pi^{-3 / 2} \exp \left(\frac{L^{\prime}\left(2 k-1 / 2, S_{y m^{2}} f\right)}{L\left(2 k-1 / 2, \text { Sym }^{2} f\right)}-\frac{3}{2} \gamma+\frac{\pi}{4}\right)
$$

is transcendental. Further, if we assume the Schanuel's conjecture, then both

$$
\exp \left(\frac{L^{\prime}\left(2 k-1 / 2, S_{y m^{2}} f\right)}{L\left(2 k-1 / 2, \text { Sym }^{2} f\right)}-\frac{3}{2} \gamma\right) \quad \text { and } \quad \frac{L^{\prime}\left(2 k-1 / 2, S_{y m^{2}} f\right)}{L\left(2 k-1 / 2, S y m^{2} f\right)}-\frac{3}{2} \gamma
$$

are transcendental.

\section{$5 \quad$ Transcendence of Artin $L$-Functions at $s=1$}

We begin with the investigation of the nature of the residue at $s=1$ of Dedekind zeta functions. Let $K$ be a number field of degree $n$. For $\Re(s)>1$, the Dedekind zeta function of $K$ is defined as

$$
\zeta_{K}(s)=\sum_{\mathrm{a}} \frac{1}{N(\mathrm{a})^{s}},
$$

where the sum is over all integral ideals of $\mathcal{O}_{K}$, the ring of integers of $K$. This is analytic for $\Re(s)>1$, and $(s-1) \zeta_{K}(s)$ extends to an entire function with

$$
\lim _{s \rightarrow 1^{+}}(s-1) \zeta_{K}(s)=\operatorname{Res}_{s=1} \zeta_{K}(s)=\frac{2^{r_{1}}(2 \pi)^{r_{2}} h_{K} R_{K}}{\omega_{K} \sqrt{\left|d_{K}\right|}},
$$

where $r_{1}$ is the number of real embeddings, $2 r_{2}$ is the number of complex embeddings, $h_{K}$ is the class number, $R_{K}$ is the regulator, $\omega_{K}$ is the number of roots of unity in $K$, and $d_{K}$ is the discriminant of $K$.

We are interested in the possible algebraic nature of $\operatorname{Res}_{s=1} \zeta_{K}(s)$ and the regulator $R_{K}$. Interestingly, because of the presence of $\pi$, the transcendence of any one of the above does not necessarily imply the transcendence of the other unless $K$ is a totally real field. We have the following. 
Theorem 5.1 Assume the Weak Schanuel Conjecture. Let $K$ be a number field. Then both the regulator $R_{K}$ and $\operatorname{Res}_{s=1} \zeta_{K}(s)$ are transcendental.

Proof By the class number formula,

$$
\operatorname{Res}_{s=1} \zeta_{K}(s)=\frac{2^{r_{1}}(2 \pi)^{r_{2}} h_{K} R_{K}}{\omega_{K} \sqrt{\left|d_{K}\right|}}
$$

where $r_{1}$ and $2 r_{2}$ are the number of real and complex embeddings. Let $u^{(j)}$ be the $j$-th conjugate of $u \in K$ and

$$
\left\{u_{1}, u_{2}, \ldots, u_{r}\right\}
$$

be a set of generators of the ordinary unit group modulo the roots of unity. Then the regulator $R_{K}$, up to a rational multiple, is given by

$$
\left|\begin{array}{cccc}
1 & \log \left|u_{1}^{(1)}\right| & \cdots & \log \left|u_{r}^{(1)}\right| \\
\vdots & \vdots & \vdots & \vdots \\
1 & \log \left|u_{1}^{(r+1)}\right| & \cdots & \log \left|u_{r}^{(r+1)}\right|
\end{array}\right| .
$$

Clearly, by Proposition 2.3 the regulator $R_{K}$ is transcendental.

Further,

$$
\pi^{r_{2}} R_{k}=F\left(\log (-1), \log \left|u_{1}^{(1)}\right|, \ldots, \log \left|u_{r}^{(r+1)}\right|\right)
$$

where $F$ is a polynomial with algebraic coefficients whose constant term is zero. Assume that the Weak Schanuel Conjecture is true. Then by Proposition 2.3. $\operatorname{Res}_{s=1} \zeta_{K}(s)$ is necessarily transcendental.

Now, we wish to extend our study to the special values of certain Artin $L$ functions, following the work of Stark [14]. We refer to [12] for a more detailed account of Artin $L$-functions. Let $K / k$ be Galois extension of number fields with Galois group $G=\operatorname{Gal}(K / k)$. Corresponding to any finite dimensional representation $(\phi, V)$ of $G$ with character $\chi$, the Artin $L$-function is defined by

$$
L(s, \chi, K / k)=\prod_{\mathcal{P}} \operatorname{det}\left(1-\left.N(\mathcal{P})^{-s} \phi\left(\sigma_{\beta}\right)\right|_{V^{I_{\beta}}}\right)^{-1},
$$

where $\mathcal{P}$ runs over all the prime ideals in $\mathcal{O}_{k}, \beta$ is a prime ideal lying over $\mathcal{P}, I_{\beta}$ is its inertia group and $\sigma_{\beta}$ is the associated Frobenius element in the Galois group. Stark in [14] made the following conjecture.

Conjecture 5.2 (Stark) Suppose $\chi$ does not contain $\chi_{0}$ as a constituent. Then

$$
L(1, \chi, K / k)=\frac{W(\bar{\chi}) 2^{a} \pi^{b}}{\left(\left|d_{k}\right| N(f)\right)^{1 / 2}} \theta(\bar{\chi}) R(\bar{\chi})
$$

We refer to Stark's article for descriptions of the terms involved. Stark proved the above conjecture for all rational characters. We have the following assertion: 
Theorem 5.3 Assume that the Weak Schanuel Conjecture is true. Then for any rational non-trivial irreducible character $\chi, L(1, \chi, K / k)$ is transcendental.

Proof Let $\chi$ be a character as above. Then, as proved by Stark,

$$
L(1, \chi, K / k)=\frac{W(\bar{\chi}) 2^{a} \pi^{b}}{\left(\left|d_{k}\right| N(f)\right)^{1 / 2}} \theta(\bar{\chi}) R(\bar{\chi})
$$

In the expression on the right-hand side, there are two possible transcendental objects, namely $\pi^{b}$ and $R(\chi)$. But we have a description of the number $R(\chi)$. It is the determinant of an $a$ by $a$ matrix whose entries are linear forms in logarithms of absolute values of units in $K$ and its conjugate fields. For instance, when $k$ is equal to $\mathbb{O}$, the entries of this matrix are given by $c_{i j}=\sum_{\sigma \in G} a_{i j}(\sigma) \log \left(\left|\epsilon^{\sigma}\right|\right)$, where $A(\sigma)=\left(a_{i j}(\sigma)\right)$ is a representation of $G$ whose character is $\chi$, and $\epsilon$ is a Minkowski unit. Since $\log (-1)=i \pi$, the residue is the value of a polynomial of the form mentioned in Proposition 2.3 evaluated at logarithms of algebraic numbers. By the work of Brauer and Aramata, we know that for any irreducible character $\chi$ of $G$, for all $t \in \mathbb{R}, L(1+i t, \chi, K / k) \neq 0$ and hence by appealing to Proposition 2.3. we see that $L(1, \chi, K / k)$ is transcendental under the Weak Schanuel Conjecture.

In general, the Weak Schanuel Conjecture will imply the transcendence of $L(1, \chi, K / k)$ whenever Stark's conjecture is true.

\section{Transcendence of the Petersson Norm}

In this section, we discuss the transcendence of the Petersson norm of cuspidal eigenforms of weight one. Suppose that

$$
f=\sum_{n=1}^{\infty} a(n) e^{2 \pi i n z} \quad \text { and } \quad g=\sum_{n=1}^{\infty} b(n) e^{2 \pi i n z}
$$

are cusp forms on $\Gamma_{0}(N)$ of weight $k \geq 1$ and character $\chi_{N}$. The Petersson inner product of $f$ and $g$ is defined as:

$$
\langle f, g\rangle:=\iint_{\Gamma_{0}(N) \backslash \mathcal{H}} y^{k-2} f(z) \overline{g(z)} d x d y .
$$

Further, if $\langle f, g\rangle \neq 0$, then we have (see [14, Section 6]):

$$
\lim _{s \rightarrow k}(s-k) G(s)=(4 \pi)^{k}\left(2 N^{2} \Gamma(k)\right)^{-1} \pi \varphi(N)\langle f, g\rangle,
$$

where

$$
G(s):=\zeta(2 s+2-2 k) \prod_{p \mid N}\left(1-p^{-2 s-2+2 k}\right) \sum_{n \geq 1} a(n) \overline{b(n)} n^{-s} .
$$

Now let $f$ be a normalised cuspidal eigenform on $\Gamma_{0}(N)$ of weight one and character $\chi_{N}$. Then $G(s)$ has a pole at $s=1$ having a residue

$$
\operatorname{Res}_{s=1} G(s)=4 \pi^{2}\left(2 N^{2}\right)^{-1} \varphi(N)\langle f, f\rangle .
$$

We have the following. 
Theorem 6.1 Let $f$ be a normalised cuspidal eigenform on $\Gamma_{0}(N)$ of weight one and nebentypus $\epsilon$. Suppose it has rational Fourier coefficients. Then its Petersson norm $\langle f, f\rangle$ is transcendental under the Weak Schanuel Conjecture.

Proof Let

$$
G(s)=\zeta(2 s) \prod_{p \mid N}\left(1-p^{-2 s}\right) \sum_{n \geq 1}|a(n)|^{2} n^{-s} .
$$

Then, we have

$$
\operatorname{Res}_{s=1} G(s)=\lim _{s \rightarrow 1}(s-1) G(s)=2 \pi^{2} \frac{\varphi(N)}{N^{2}}\langle f, f\rangle .
$$

By a theorem of Deligne and Serre [5], there exists an irreducible representation

$$
\rho: \operatorname{Gal}(K / \mathbb{O}) \rightarrow \mathrm{GL}(2, \mathbb{C})
$$

of conductor $N$ and determinant $\epsilon$ whose Artin $L$-function is

$$
L(s, \chi, K / \mathbb{O})=\sum_{n=1}^{\infty} a(n) n^{-s} .
$$

Here $\chi$ is the character of $\rho$. Then $\chi \bar{\chi}$ is of degree 4 and the corresponding Artin $L$-function is given by

$$
L(s, \chi \bar{\chi}, K / \mathbb{Q}))=\prod_{p \mid N}\left(1-p^{-s}\right)^{-1} G(s) .
$$

Hence, we have

$$
\langle f, f\rangle=\frac{N}{2 \pi^{2}} \lim _{s \rightarrow 1}(s-1) L(s, \chi \bar{\chi}, K /(\mathbb{O}) .
$$

Since $\psi=\chi \bar{\chi}-1$ is rational valued, by Stark, we have:

$$
L\left(1, \psi, K /(\mathbb{O})=\frac{W(\bar{\psi}) 2^{a} \pi^{b}}{(N(f))^{1 / 2}} \theta(\bar{\psi}) R(\bar{\psi}) .\right.
$$

Here other than $\pi$ and the regulator $R(\bar{\psi})$, all other terms are algebraic. For complex numbers $A$ and $B$, let us denote by $A \sim B$ when $A$ is an algebraic multiple of $B$. Thus,

$$
L(1, \psi, K / \mathbb{O}) \sim \pi^{b} R(\bar{\psi}) .
$$

Now $L(s, \chi \bar{\chi}, K / \mathbb{Q})$ factors as the product of $L(s, \psi, K / \mathbb{O})$ and a Dedekind zeta function $\zeta_{k}(s)$. Since

$$
\lim _{s \rightarrow 1^{+}}(s-1) \zeta_{k}(s)=\operatorname{Res}_{s=1} \zeta_{k}(s)=\frac{2^{r_{1}}(2 \pi)^{r_{2}} h_{k} R_{k}}{\omega_{k} \sqrt{\left|d_{K}\right|}},
$$

we have $\langle f, f\rangle \sim \pi^{b-2+r_{2}} R(\bar{\psi}) R_{k}$ by (6.1). But as noted before, the terms $R(\bar{\psi})$ and $R_{k}$ are determinants of matrices whose entries are linear forms in logarithms of algebraic numbers. Hence by Proposition 2.3, we note that $\langle f, f\rangle$ is transcendental. 
We now consider some explicit cases where the transcendence of the Petersson norm can be deduced unconditionally.

Let

$$
S=\{23,31,59,83,107,139,283,307,331,379,499,547,643,883,907\}
$$

For $N \in S$, let us consider the quadratic extension $k=(0)(\sqrt{-N})$. The class number of $k$ is 3 . Let $K$ be the Hilbert class field of $k$. Then the Galois group $G$ of $K / \mathbb{O})$ is $S_{3}$. Then $G$ has a two dimensional irreducible character $\chi$ that is rational valued. Let

$$
L(s, \chi, K / \mathbb{O}))=\sum_{n \geq 1} a(n) n^{-s}
$$

be the Artin $L$-function associated with $\chi$. By the works of Langlands and Tunnell [17], such an $L$-function is modular. Thus,

$$
f:=\sum_{n=1}^{\infty} a(n) e^{2 \pi i n z}
$$

is a cusp form of weight 1 on $\Gamma_{0}(N)$. As before,

$$
\langle f, f\rangle=\frac{N}{2 \pi^{2}} \lim _{s \rightarrow 1}(s-1) L(s, \chi \bar{\chi}, K /(\mathbb{O}) .
$$

In this case, the $L(s, \chi \bar{\chi}, K /(\mathbb{O})$ factors as

$$
L\left(s, \chi \bar{\chi}, K /(\mathbb{Q})=\zeta_{k}(s) L(s, \chi, K /(\mathbb{Q})\right.
$$

and thus

$$
\left.\langle f, f\rangle=\frac{N}{2 \pi^{2}} \frac{3 \pi}{\sqrt{N}} L(1, \chi, K /(\mathbb{O}))=\frac{3 \sqrt{N}}{2 \pi} L(1, \chi, K / \mathbb{O})\right)
$$

Substituting the expression for $L(1, \chi, K / \mathbb{O}))$ from Stark's theorem, we have

$$
\langle f, f\rangle=\frac{3 \sqrt{N}}{2 \pi} \frac{2 \pi}{\sqrt{N}} \theta(\bar{\chi}) W(\bar{\chi}) R(\bar{\chi}) \sim R(\bar{\chi}) .
$$

In this case, $R(\bar{\chi})=\log \epsilon$, where $\epsilon$ is the Minkowski unit. Thus, by the classical theorem of Hermite and Lindemann, $\langle f, f\rangle$ is transcendental.

Remark 6.2 Let $f$ be a normalised weight one cuspidal eigenform on $\Gamma_{0}(N)$ with nebentypus $\epsilon$. Then using the Deligne-Serre theorem and arguing as before, we see that if Stark's conjecture is true, $\langle f, f\rangle$ is transcendental under Schanuel's conjecture.

Acknowledgment We are grateful to Professor M. Waldschmidt for going through an earlier version of our paper. 


\section{References}

[1] S. D. Adhikari, N. Saradha, T. N. Shorey, and R. Tijdeman, Transcendental infinite sums. Indag Math. 12(2001), no. 1, 1-14. doi:10.1016/S0019-3577(01)80001-X

[2] A. Akbary, Non-vanishing of weight $k$ modular L-functions with large level. J. Ramanujan Math. Soc. 14(1999), no. 1, 37-54.

[3] C. Breuil, B. Conrad, F. Diamond, and R. Taylor, On the modularity of elliptic curves over $(0)$ : wild 3-adic exercises. J. Amer. Math. Soc. 14(2001), no. 4, 843-939. doi:10.1090/S0894-0347-01-00370-8

[4] B. Conrad, F. Diamond, and R. Taylor, Modularity of certain potentially Barsotti-Tate Galois representations. J. Amer. Math. Soc. 12(1999), no. 2, 521-567. doi:10.1090/S0894-0347-99-00287-8

[5] P. Deligne and J.-P. Serre, Formes modulaires de poids 1. Ann. Sci. École Norm. Sup. (4) 7(1974), 507-530 (1975)

[6] F. Diamond, On deformation rings and Hecke rings. Ann. of Math. (2) 144(1996), no. 1, 137-166. doi:10.2307/2118586

[7] S. Gun, M. Ram Murty, and P. Rath, Transcendence of the log gamma function and some discrete periods. J. Number Theory 129(2009), no. 9, 2154-2165. doi:10.1016/j.jnt.2009.01.008

[8] L Linear independence of digamma function and a variant of a conjecture of Rohrlich. J. Number Theory 129(2009), no. 8, 1858-1873. doi:10.1016/j.jnt.2009.02.007

[9] S. Lang, Introduction to modular forms. Grundlehren der mathematischen Wissenschaften, 222, Springer-Verlag, Berlin-New York, 1976.

[10] Introduction to transcendental numbers. Addison-Wesley Publishing Co., Reading, Mass.-London-Don Mills, Ont. 1966.

[11] M. Lerch, Dalsi studie v oboru Malmstenovskych rad, Rozpravy Ceske Akad. 18(1894), no. 3, 63pp.

[12] M. Ram Murty, An introduction to Artin L-functions. J. Ramanujan Math. Soc. 16(2001), no. 3, 261-307.

[13] M. Ram Murty and N. Saradha, Transcendental values of the digamma function. J. Number Theory 125(2007), no. 2, 298-318. doi:10.1016/j.jnt.2006.09.017

[14] H. M. Stark, L-functions at s = 1. II. Artin L-functions with rational characters. Advances in Math. 17(1975), no. 1, 60-92. doi:10.1016/0001-8708(75)90087-0

[15] G. Shimura, On the holomorphy of certain Dirichlet series. Proc. London Math. Soc. (3) 31(1975), no. 1, 79-98. doi:10.1112/plms/s3-31.1.79

[16] R. Taylor and A. Wiles, Ring-theoretic properties of certain Hecke algebras. Ann. of Math. (2) 141(1995), no. 3, 553-572. doi:10.2307/2118560

[17] J. Tunnell, Artin's conjecture for representations of octahedral type. Bull. Amer. Math. Soc. (N.S.) 5(1981), no. 2, 173-175. doi:10.1090/S0273-0979-1981-14936-3

[18] L. C. Washington, Introduction to cyclotomic fields. Graduate Texts in Mathematics, 83, Springer-Verlag, New York, 1982.

[19] M. Waldschmidt, Diophantine approximation on linear algebraic groups. Transcendence properties of the exponential function in several variables. Grundlehren der Mathematischen Wissenschaften, 326, Springer-Verlag, Berlin, 2000.

[20] A. Wiles, Modular elliptic curves and Fermat's last theorem. Ann. of Math. (2) 141(1995), no. 3, 443-551. doi:10.2307/2118559

[21] D. Zagier, Values of zeta functions and their applications. In: First European Congress of Mathematics, Vol. II (Paris, 1992), Progr. Math., 120, Birkhäuser, Basel, 1994, pp. 497-512.

The Institute of Mathematical Sciences, CIT Campus, Taramani, Chennai 600113, India e-mail:sanoli@imsc.res.in

Department of Mathematics, Queen's University, Kingston, ON K7L 3N6 e-mail: murty@mast.queensu.ca

Chennai Mathematical Institute, Padur PO, Siruseri 603103, Tamilnadu, India

e-mail: rath@cmi.ac.in 